\title{
Deep Learning for Ground Reaction Force Data Analysis Application to Wide-Area Floor Sensing
}

DOI:

10.1109/ISIE.2019.8781511

\section{Document Version}

Accepted author manuscript

Link to publication record in Manchester Research Explorer

\section{Citation for published version (APA):}

Alharthi, A., \& Ozanyan, K. (2019). Deep Learning for Ground Reaction Force Data Analysis Application to WideArea Floor Sensing. In The 28th International Symposium on Industrial Electronics (ISIE) (pp. 1401-1406). [978-17281-3666-0/19/\$31.00 @2019 IEEE] IEEE. https://doi.org/10.1109/ISIE.2019.8781511

\section{Published in:}

The 28th International Symposium on Industrial Electronics (ISIE)

\section{Citing this paper}

Please note that where the full-text provided on Manchester Research Explorer is the Author Accepted Manuscript or Proof version this may differ from the final Published version. If citing, it is advised that you check and use the publisher's definitive version.

\section{General rights}

Copyright and moral rights for the publications made accessible in the Research Explorer are retained by the authors and/or other copyright owners and it is a condition of accessing publications that users recognise and abide by the legal requirements associated with these rights.

\section{Takedown policy}

If you believe that this document breaches copyright please refer to the University of Manchester's Takedown Procedures [http://man.ac.uk/04Y6Bo] or contact uml.scholarlycommunications@manchester.ac.uk providing relevant details, so we can investigate your claim.

\section{OPEN ACCESS}




\section{Deep Learning for Ground Reaction Force Data Analysis: Application to Wide-Area Floor Sensing}

\author{
Abdullah S Alharthi \\ School of Electrical and Electronic Engineering \\ The University of Manchester, United Kingdom \\ Abdullah.alharthi@manchester.ac.uk
}

\begin{abstract}
Deep learning methods are proposed to process and fuse raw spatiotemporal ground reaction forces (GRF) to accurately categorize gait pattern. These methods are based on convolutional neural network and long short-term memory networks architectures to learn spatiotemporal features, automatically end-to-end from raw GRF sensor signals. In a case study on Parkinson's disease (PD) data, spatiotemporal signals of gait for PD patient and healthy subjects are processed and classified, resulting an effective gait pattern classification with a precision performance of $96 \%$. Deep learning considerably achieved better classification results, compared to the shallow learning methods with the handcrafted features. This implies that for the purpose of automatic decision-making, it is beneficial to utilize deep learning methods to analyse GRF. This insight is portable across a range of industrial tasks that involve complex spatiotemporal GRF signals classification. The proposed models are computationally efficient and able to achieve high classification precision from a large set of GRF signals.
\end{abstract}

Keywords-Convolutional Neural Networks (CNN), Deep learning, Floor sensing, Ground Reaction Forces (GRF), Gait, Long Short-Term Memory Networks (LSTM), Parkinson's disease

\section{INTRODUCTION}

In recent years, intelligent chips, electronics, and sensing technologies have fostered substantial interest in activity recognition for commercialized applications such as smartphone, smartwatches, and health monitoring devices. Much of that success has dual-use applications outside wearables and body area networks, e.g. in occupancy monitoring and identification of object dynamics on a busy factory floor, with clear relevance to health and safety. Furthermore, in the effort to develop the nascent potential for a wide societal roll-out of anthropomorphic robotics, safe and adequate human-robot interaction will be aided by floor-based ground-reaction force (GRF) data, as an essential part of multimodality sensor fusion.

The development of analytical computing technologies presents new opportunities for processing, detecting, and classifying different multi-source multi-sensor data. Deep learning allows to automatically produce models that can analyse more significant, complex GRF data and deliver faster, more accurate results even on very large datasets.

For such a data-centric approach it is unrealistic to expect rapid progress where data is not available in the required size and quality; thus, piloting such methodology depends on accessing other areas of research where comparable problems can be defined and solutions sought on data which are available

\author{
Krikor B Ozanyan \\ School of Electrical and Electronic Engineering \\ The University of Manchester, United Kingdom \\ k.ozanyan@manchester.ac.uk
}

and well understood. In support of such an approach, a variety of commercialized healthcare applications have attracted attention due to the possibility of using gait spatiotemporal parameters at the basis for gait anomaly detection devices. In this work, the performance of deep neural networks is studied with a view to achieving the required sensitivity for accurate classifications from GRF data. Due to lacking data alternatives at present, we use human gait GRF data captured by a pressure sensor under the foot. Although the models trained with such particular data will not be identical in all GRF application cases, the spatio-temporal character of the data, as well as the task to extract classification features automatically, remain the same.

Progress in instrumentation for GRF sensing in human gait has given rise to the objective evaluation of different human locomotion parameters. It has included, but not been limited to, commercialized devices for detection and monitoring of abnormal gait. Due to the complex nature of the gait cycle, presenting and interpreting a result from gait spatio-temporal attributes in real-world scenarios, such as healthcare where clinical verification is possible, would be a strong steer as to what to expect from deep learning in other GRF-related cases.

We use the case of Parkinson's disease (PD) as a neurodegenerative disorder that causes muscular stiffness, tremor, soft voice, shuffling gait, slowness of the movement, poor postural stability, freezing of gait [1]. Typically, an expert doctor examines PD patients depending on established criteria to rate the severity of the disease, the rating criteria: Unified PD Rating Scale, Hoehn and Yahr staging, and the Schwab and England rating of activities of daily living [2]. Abnormal gait is one of the critical features of PD, and monitoring a patient while he walks is probably the most valuable procedure in the diagnostic workup of PD. However, in the early stages of PD, gait evaluation may lead to inconclusive results because of slow walking and short stride are often doubtful and can be related to age, depressive mood, or other conditions [3].

It is evident that foot placement and the resulting GRF sequence of a healthy person is different from PD patients. The healthy person would have a pronounced heel strike and stepping off with their toes, then landing on their heel again, while PD patients tend to have a flat foot in the strike profile [4]. Heel strike is one of the gait cues and it can be picked up to recognize $P D$, as well as rate the severity of the deviation from normal. Essentially sensors under the foot are suitable to capture spatiotemporal information of the heel strike patterns while walking. However, to commercialize sensors for abnormal gait recognition in healthcare, as well as for 
monitoring the dynamics in the floor occupancy by a number of different players, it is necessary to integrate in their design the appropriate sub-systems to process the complex GRF spatiotemporal information. Integrated sensor-actuator systems, such as smart prosthetics or biped robots with adaptive locomotion, would require deep learning for solving the complex problems of capturing the GRF data and test the validity of the training, before implementing it on a hardware to be used as standalone system to assist a real-time factory floor management or as a tool in aid for PD severity rating.

Various machine learning methods have been proposed to categorize the complex spatiotemporal GRF character. To classify gait as normal or PD, [5] proposed Shifted 1D- Local Binary Patterns (LBP) to extract features and apply Multi-Layer Perceptron (MLP) for the diagnosing of PD. In [6] a statistical learning, principal component analysis (PCA) is proposed to classify the data and identify the presence of PD. Thus, the salient features of spatiotemporal GRF may be lost in the process of feature extraction, producing lower accuracy. This can be mitigated by utilizing deep learning methods for its capability of automatic feature extraction, delivering high statistical confidence by fusing sensors data and learn rich features of gait patterns in the neural network deep layers.

We propose a convolutional neural network (CNN) and a long short-term memory neural network (LSTM) to learn sensors spatiotemporal information from raw GRF signals, to recognize the features associated with gait disorders in PD. The GRF recognition algorithms are based on deep learning methods to fuse sensors signals and extract gait feature automatically from GRF signature in minimal time and with extremely light and fast computing ability. Furthermore, the models are extremely versatile to personalize their owner's experience on gait feature.

\section{BACKGROUND}

Gait is a behavioural recurrent contact with the surface to move from one position to the other in humans, and it is achieved through the synchronised movement of the lower limbs [7]. Gait analysis is an active research area for a variety of applications including sport [8]-[9], identification of individuals for security [10]-[11], and healthcare [12]-[13].

Gait is obtained to study the potentials of footsteps in the analysis of gait variability to study the cognitive decline.
Freezing of gait (FOG) is a disorder in late stage Parkinson's disease which causes fall, and loss of independents have been investigated using electroencephalography (EEG) data while participants are stepping on a force plate. The participants EEG have been studied to rate the FOG severity by analysing the signals during gait cycle [14]. Lorenzi et al. [15] used a single IMU unit positioned on the head, to collect gait patterns during the gait cycle, aiming to distinguish normal gait from the freezing of gait and irregular steps in Parkinson's disease (PD), using dynamic time warping to select the input features to the ANN. Abdulhay [4] presented a study on detecting Parkinson's disease (PD) based on ground reaction force of individuals during gait cycle. The study shows that stance time, swing time, stride time and foot strike profile can be used to distinguish PD patients from a normal person.

\section{PAtTERn ReCOGNITION MEthods}

Supervised learning is by far the most widely used machine learning method in gait analysis. The main objective of the proposed algorithms is to find the hypothesis to our desired PD ground truth labels provided with the input. Since the difference between the gait cycle of PD patient and normal person is unknown, deep learning algorithms is utilized to correctly map the sensed gait spatiotemporal information to a ground truth label, which is normal or PD with Hoehn and Yahr staging. In this process the algorithms can easily learn by itself the events occur during gait cycle. Further, the methods are tested with unseen data to measure the models accuracy. The validity of this innovative approach to the problem of gait GRF signal processing and sensor fusion in the neural network deep layers is demonstrated by performing calculations outboard with a PC using Python Keras open source library [16].

\section{A. CNNs Network}

State of the art deep convolutional neural network is the most favorable method for data that has a known grid-like topology [17]. The CNNs learns a high level of abstraction and pattern from large datasets by applying to the input convolution operations. Commonly, the network consists of convolution layers, pooling layers and normalization layers, with a set of filters and weights shared among these layers. Convolution operation given an input $I(t)$ and a kernel $K(a)$ is given as:

$$
S(t)=\sum_{a} I(a) * K(t-a)
$$

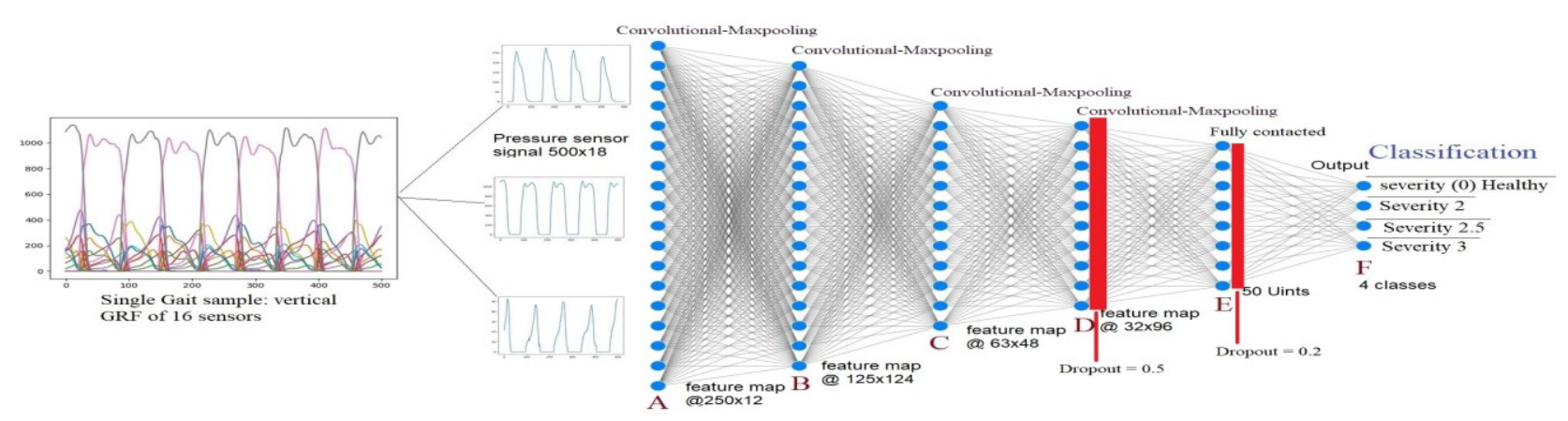

Fig. 1. Illustration of the proposed deep 1D-CNNs architecture. (A): Input-layer; (B): Hidden-layer; (C): Hidden-layer; (D): Hidden-layer (E): Fully contacted-layer; (F): Fully contacted-output layer. 


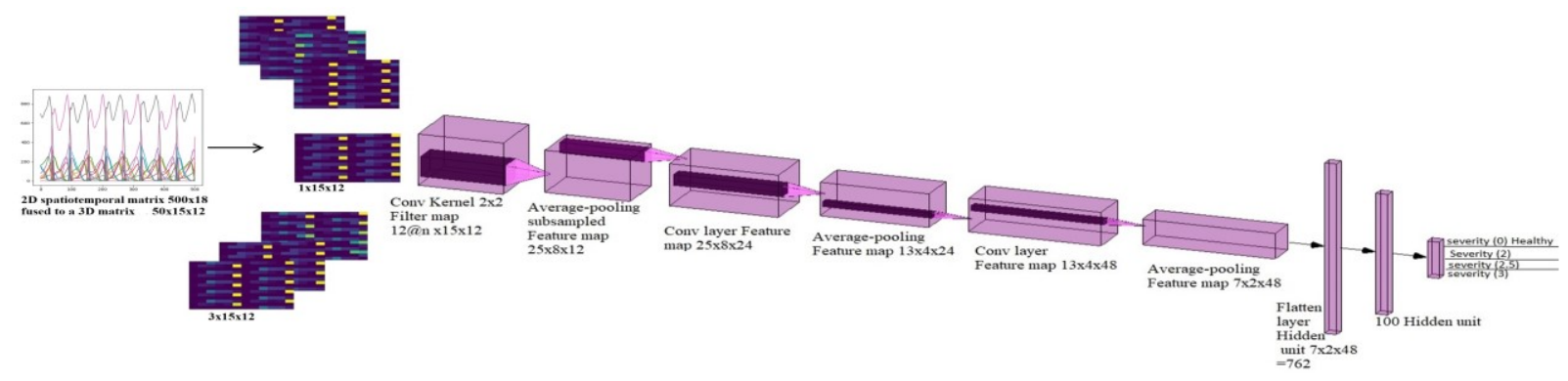

Fig. 2. Illustration of the proposed deep 2D-CNNs architecture

Tow CNNs neural networks are propose based on the input as follow:

\section{a) $1 D-C N N$}

A deep 1D-CNNs model (See architecture in figure 1) is built to automatically extract gait feature directly from the raw spatiotemporal sensor signals. The network consists of four convolutional layers each followed by Max pooling and two fully connected layers, a total of nine stacked layers. The first convolutional layer takes as input raw spatio-temporal GRF signals matrix. A stride of 1 and same-padding used in the convolutional layers to output the same length of the original input shape, followed by Max-pooling layer to subsample the feature map and make the convolutional layer output more robust.

\section{b) $2 \mathrm{D}-\mathrm{CNN}$}

The network input is fused spatio-temporal $3 \mathrm{D}$ matrix of the raw sensor signals [18]. The network consists of three convolutional layers each followed by an average pooling and two fully connected layers (See architecture in figure 2) a total of seven stacked layers. The first convolutional layer takes as input fused spatio-temporal signals volume of 50 with 15 (height) by 12 (width) spatial dimensions. The four convolutional layers have $n$ channels each that assign one frame of the input to a single convolutional layer channel. The convolutional layers use a stride of 1 , same-padding, and a filter of $2 \times 2$ dimensions for the average-pooling layers.

\section{B. LSTM Network}

The LSTM neural network is favourable for processing time series data, where the order is of importance. Since gait cycle is time related cue, the LSTM approach is found to be quite useful in gait data analysis. We propose LSTM network with five stacked layers, where the first and second layer have memory

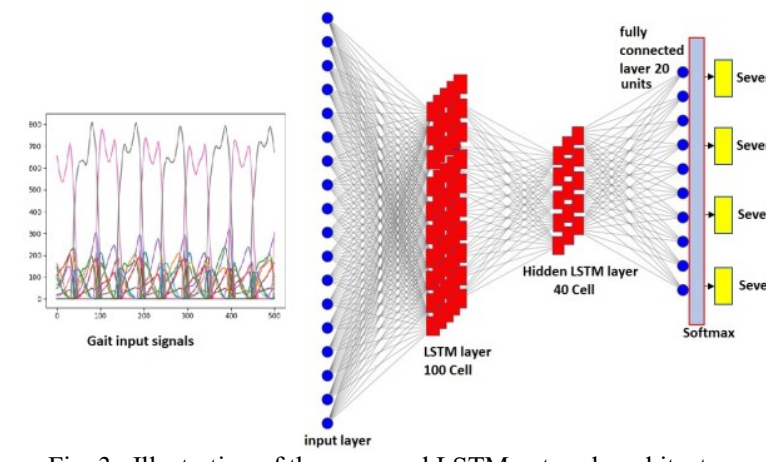

Fig. 3. Illustration of the proposed LSTM network architecture blocks. Instead of neurons, the blocks are connected together in each layer. Each block contains Forget Gate to control which data remains in the block, Input Gate to control which data flow to the block, and Output Gait to control the output based on the computed activation in the block. The last three layers are fully connected layer, batch normalization layer, and a fully connected output layer. An illustration of the LSTM architecture is shown in figure 3 .

\section{Shallow Learning Methods}

The shallow learning is the classical way to perform classification based on gait handcrafted features to learn in predefined relationship to classify the inputs to the output ground truth. Four shallow learning methods are utilized to compare the performance with the proposed deep learning methods. The used shallow learning methods are: decision tree classifier, logistic regression, support vector machine (SVM), and multilayer perceptron neural network with input layer and output fully connected layer.

\section{Dataset}

Open access benchmark created by research group supported by National Institutes of Health, the National Parkinson Foundation, and the Parkinson's Disease Foundation, is obtained to train and test the algorithm. The dataset is acquired from PhysioNet and cleaned for processing. It consists of 93 PD patients (mean age: 66.3 years; 63\% men) (See table I) and with different level of the disease (See table II) and 73 healthy control (mean age: 66.3 years; 55\% men). The dataset consists of a vertical ground reaction force of subjects collected as they walked for approximately two minutes. Each subject had eight

Table. I

Subject's dataset discerption

\begin{tabular}{ccccc}
\multicolumn{5}{c}{ Subject's dataset discerption } \\
\hline Subjects & \# subjects & Male & Female & Group \\
\hline PD patients & 29 & 20 & 9 & Ga [19] \\
Healthy Subjects & 18 & 10 & 8 & Ga [19] \\
PD patients & 29 & 16 & 13 & Ju [20] \\
Healthy Subjects & 26 & 12 & 14 & Ju [20] \\
PD patients & 35 & 22 & 13 & $\mathrm{Si} \mathrm{[21]}$ \\
Healthy Subjects & 29 & 18 & 18 & $\mathrm{Si}[21]$ \\
\hline
\end{tabular}

Table. II

Number of subjects with the severity rating

\begin{tabular}{ccccc}
\multicolumn{5}{c}{ Number of subjects with the severity rating } \\
\hline $\begin{array}{c}\text { severity } \\
(0)\end{array}$ & $\begin{array}{c}\text { severity } \\
(2)\end{array}$ & $\begin{array}{c}\text { severity } \\
(2.5)\end{array}$ & $\begin{array}{c}\text { severity } \\
(3)\end{array}$ & Group \\
Healthy & & & & \\
\hline 18 & 15 & 8 & 6 & $\mathrm{Ga}[19]$ \\
26 & 12 & 13 & 4 & $\mathrm{Ju}[20]$ \\
29 & 29 & 6 & 0 & $\mathrm{Si} \mathrm{[21]}$ \\
\hline
\end{tabular}


sensors underneath each foot to capture the forces placed on the ground as a function of time. The output of the 16 sensors total of two feet is recorded as 100 frames per second. Also, the sum of the eight sensors of each foot is added to each subject sample. The data set is collected by three gropes namely Ga group [19], $\mathrm{Ju}$ group [20] and Si group [21]. Where group Ga included additional sample for each subject, which is performing serial7 subtraction while walking. Group $\mathrm{Ju}$ and $\mathrm{Si}$ recorder usual normal walk with self-selected speed.

\section{EXPERIMENT}

\section{A. Data Preprocessing}

The dataset samples are downloaded from PhysioNet Gait in Parkinson's Disease, as a text file per sample. Each text file is converted to a python file. Each sample is 19 columns, some of which have 12119 frames and the other have 1000 frames persample. In order to make the dataset the same length, the datasets were divided to equal size of 500 frames and the timestamp columns were deleted. The final sample size is 18 columns and 500 rows or frames. This method is adapted since the gait cycle is one second and the sample will include both feet heel strike and toe off for five gait cycles. The final dataset is a tensor with the following dimensional $\mathrm{K} \times 500 \times 18$ where $\mathrm{K}=2698$ for $\mathrm{Ga}$ group, 2198 for $\mathrm{Ju}$ and $1509 \mathrm{Si}$ group (See figure 4). Data standardization is performed as a pre-processing step. Standardization is an essential step to reduce the redundancy and dependency among the data to make it suitable for machine learning to perform classification. The standardization involves rescaling the distribution of values by removing the mean and rescaling the standard deviation to unity. Next, the dataset was split into training $80 \%$ and testing $20 \%$ with a random state of 42.

\section{B. Shallow Learning}

Supervised shallow learning algorithms are implemented to perform classification using the ground truth provide with the input. Features are extracted from GRF raw signals, special

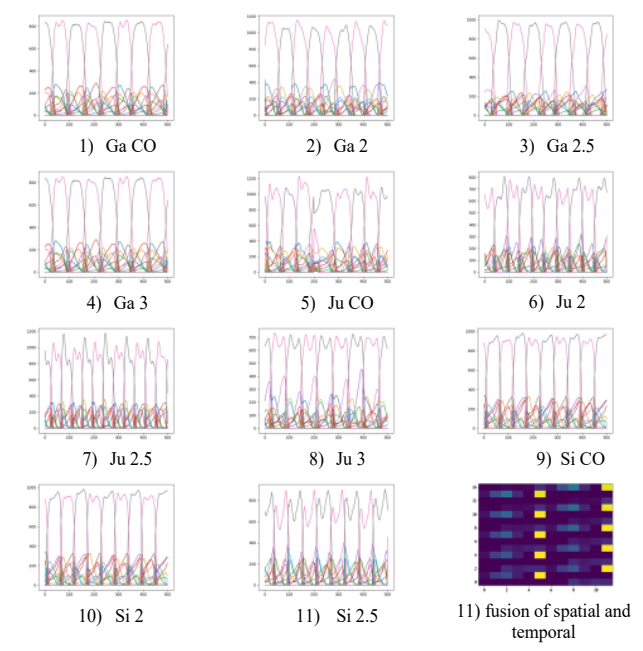

Fig. 4. Data samples from each group 1-10, CO is a normal subject sample and 2, 2.5, 3 are the PD severity levels; 11) spatiotemporal fusion matrix $15 \times 12$ average and standard deviation [22] is adapted to extract the features.

\section{a) Spatial average}

The spatial average feature takes the average of all the sensor signals $\mathrm{R}$ at each timestamp t. The spatial average is calculated as follows:

$$
S A[t]=\frac{1}{18} \sum_{i=1}^{18}\left(R_{i}[t]\right)
$$

\section{b) Standard deviation}

The standard deviation feature calculates the spread of the distribution of the intensity values coming from all the sensor signals at each time step. The standard deviation feature is expressed as follows:

$\phi[t]=\sqrt{\frac{1}{18}\left[\left(\left(R_{1}-S A[t]\right)\right)^{2}+\left[\left(\left(R_{2}-S A[t]\right)\right)^{2}+\cdots+\left[\left(\left(R_{18}-S A[t]\right)\right)^{2}\right.\right.\right.}$

\section{Deep Feature Learning and Classifications}

\section{a) CNNs training and testing}

The models are trained and validated using a patch size of 180 samples for each iteration, 100 echoes are optimal to train the models. The training size is $80 \%$ of the training data and $20 \%$ for validation. An Adam (A Method for Stochastic Optimization) [23] is used to train the model. The optimizer parameters are adjusted as follow: $\alpha=0.002, \beta 1=0.9, \beta 2$ $=0.999, \varepsilon=1 \mathrm{e}-08$. Where $\alpha$ is the learning rate or the proportion that weights are updated. $\beta 1$ is the exponential decay rate for the first moment estimates. $\beta 2$ is the exponential decay for the second-moment estimates. $\varepsilon$ is a small number to avoid any division by zero in the implementation. The loss is computed using categorical cross-entropy in every iteration. To improve the model's performance a regularization method is utilized, a dropout with a size of 0.5 was added after the last MaxPooling layer was flattened and an additional dropout with the size of 0.2 was added before the output layer in the $1 \mathrm{D}-\mathrm{CNN}$ model, and a dropout of size 0.5 was added after the last MaxPooling layer was flattened in the 2D-CNN model. The model's hyperparameters are selected based on extensive experimentation are shown in table III.

\section{b) Deep-LSTM training and testing}

The model is trained and validated using a patch size of 200 samples for each iteration, 100 echoes are optimal to train the model. The same optimization in (IV.C.a) is used to train and validate the model. The LSTM hyper-parameters are as follow: first LSTM layer has 100 units and the second LSTM layer has 40 units with a dropout of 0.2 and recurrent dropout of 0.2 , followed by Fully- connected layer with 20 units, the top output Fully-connected layer has a softmax activation function to produce four classes of PD severity levels. To improve the model performance, we add a Batch Normalization followed by a dropout with a size of 0.5 after the LSTM hidden layer.

\section{RESUlts}

This section presented the performance of the shallow and deep learning algorithms to classify PD severity levels. The confusion matrix methods is adapted [24] to evaluate the 
Table. III CNN hyper-parameters

\begin{tabular}{|c|c|c|c|c|c|c|c|}
\hline \multicolumn{4}{|l|}{$\mathrm{A}$} & \multicolumn{4}{|l|}{ B } \\
\hline Model Layers 1D-CNN & 1D-Filter & Activation & $\begin{array}{l}\text { Layer Output } \\
\text { 1D-CNN }\end{array}$ & Model Layers 2D-CNN & 2D-Filter & Activation & $\begin{array}{r}\text { Layer Output 2D- } \\
\text { CNN }\end{array}$ \\
\hline Conv1 & 12 & ReLU & $500 \times 12$ & 2D-Conv1 & 12 & ReLU & $50 \times 15 \times 12$ \\
\hline Max-pooling & 2 & - & $250 \times 12$ & 2DAverage-Pooling & 2 & - & $25 \times 8 \times 12$ \\
\hline Conv2 & 24 & ReLU & $250 \times 24$ & 2D-Conv2 & 24 & ReLU & $25 \times 8 \times 24$ \\
\hline Max-pooling & 2 & - & $125 \times 24$ & 2D-Average-Pooling & 2 & - & $13 \times 4 \times 24$ \\
\hline Conv3 & 48 & ReLU & $125 \times 48$ & 2D-Conv3 & 48 & ReLU & $13 \times 4 \times 48$ \\
\hline Max-pooling & 2 & - & $63 \times 48$ & 2D-Average-Pooling & 2 & - & 7248 \\
\hline Conv4 & 96 & ReLU & $63 \times 96$ & Fully-connected & 100 & ReLU & 100 \\
\hline Max-pooling & 2 & - & $32 \times 96$ & Fully-connected output & 4 & softmax & 4 \\
\hline Fully-connected & 50 & ReLU & 50 & & & & \\
\hline Fully-connected output & 4 & softmax & 4 & & & & \\
\hline
\end{tabular}

A) 1D-CNN; B) 2D-CNN

proposed methods for its ability to deliver accurate classification results. Figure 5 shows the proposed algorithms performances.

Figure 6 illustrates four shallow learning algorithms performance with two hand crafted features. The SVM achieved lower accuracy compared to the other algorithms. The highest accuracy is $90 \%$ achieved by the decision tree classifier using the standard deviation input.

Figures: 7, 8, 9, shows the classification result on four classes of healthy and PD with severity levels. In figure 7, 1DCNNs confusion matrix shows the model performance on classifying PD severity with $95 \%$ accuracy, recall, precision, and F1 score. The model loss is less than 4\% in training and validation process which is a good fit model. Further, training and validation are completed in 11.47 minutes. This is due to

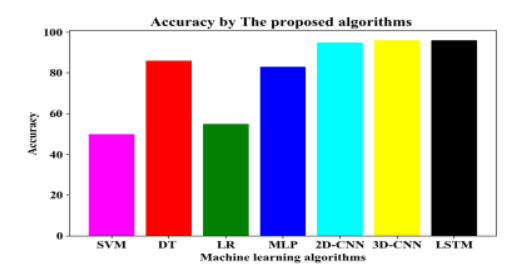

Fig. 5. A comparison among the proposed algorithms

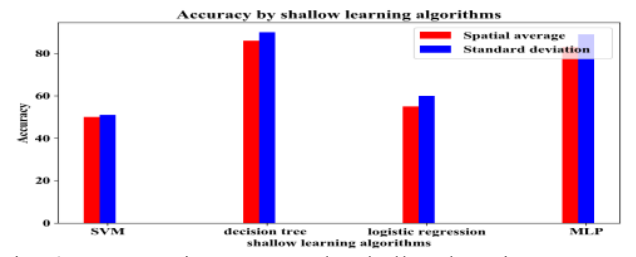

Fig. 6. A comparison among the shallow learning accuracv the dataset large size, the total GRF signals for training and validation is 46116000 signals.

The 2D-CNN model confusion matrix is shown in figure 8 . According to the results, the model classified the PD severity with $96 \%$ accuracy, recall, precision, and F1 score. Severity level 2 has the lowest accuracy, scoring 93\%. The model loss is less than $3 \%$ in training and validation process. The training and validation time is 9.47 minutes.

LSTM model confusion matrix resulted in a classification of PD severity with $96 \%$ accuracy, recall, precision, and F1 score, and the lowest classification score was on severity level 3 as shown in figure 9 . However, the training and validation time is 90 minutes. This is basically due to the large dataset's size and the memory blocks capabilities in processing time series data.

Further, the proposed deep learning methods outperformed machine learning methods proposed in [4],[5], [6],[25] on the three datasets as shown in table IV to classify subjects GRF as control or PD.

\section{DISCUSSION AND CONCLUSIO}

The character of gait GRF data poses the problem of identifying features suitable for gait classifications, desirable in a number of industrial application areas. Due to the complex set of spatio-temporal attributes in real-world gait scenarios, we use a case study as a steer to strategies in other GRF-related areas, such as the interaction of machines/robots and humans in industrial settings.

Deep learning methods are proposed to extract gait feature from GRF signals to classify abnormal gait associated with Parkinson's disease. The clinically defined severity levels based on ground truth diagnostic is rated by $96 \%$. We used

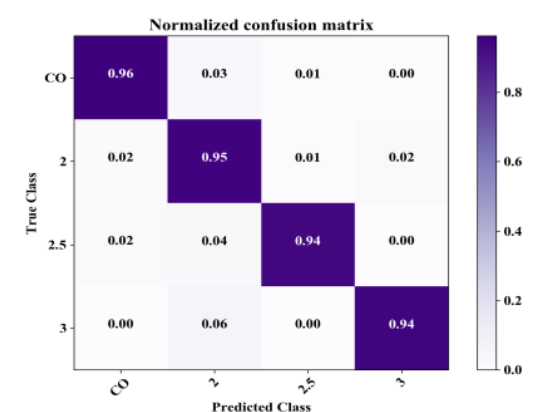

Fig. 7. 1D-CNNs confusion matrix classification results

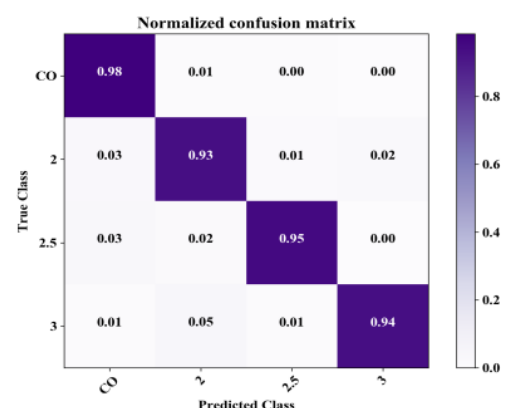

Fig. 8. 2D-CNNs confusion matrix classification results

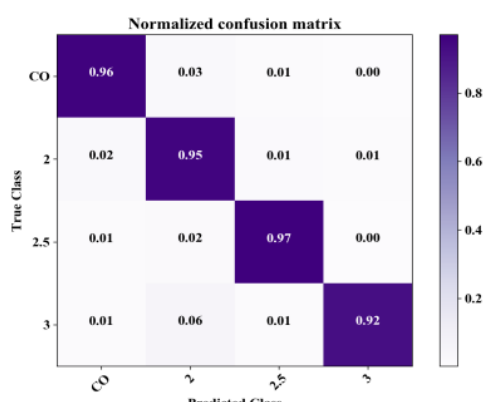

Fig. 9. LSTM confusion matrix classification results 
Table. IV PD classification results on PhysioNet three datasets

\begin{tabular}{ccc}
\hline Reference & Methods & Accuracy \\
\hline E. Abdulhay et al. [4] & SVM & $92.7 \%$ \\
Ertuğrul et al. [5] & 1D-LBP+MLP & $88.89 \%$ \\
Medeiros et al. [6] & PCA & $81.00 \%$ \\
Wu et al. [25] & SVM & $84.48 \%$ \\
Our methods & 2D-CNN \& LSTM & $\mathbf{9 6 . 0 0 \%}$ \\
\hline
\end{tabular}

public datasets" PhysioNet", which consist of Parkinson's disease patients and healthy control subjects GRF signals to test the validity of the algorithms. To demonstrate the robustness of the proposed algorithms, the three datasets are combined to rate PD severity. Deep CNN and LSTM methods achieved high accuracy in processing and fusing GRF signals. These innovative approaches reflect in extremely light and fast computation on large datasets. 2D-CNN model achieved high precision with less computational time on a complex 3D tensor of GRF signals. Based on the high precision, the proposed deep learning methods outperformed the proposed approaches reported in the literature, additionally our methods are capable of classifying multi-category gait GRF to rate PD severity levels.

At this point of research, the methodologies were implemented and tested with a PC. In the final stage, the developed models will be loaded into a microcontroller unit to perform real-time signal processing directly on board for fast computing. In future work, a fusion in the score level of CNN and LSTM will be implemented to take advantage of the CNN in recognizing the pattern and the LSTM internal memory for processing time series data to achieve higher accuracy in processing GRF signals.

\section{REFERENCES}

[1] S. Fahn et al. "Levodopa and the progression of Parkinson's disease," The New England Journal of Medicine. vol. 351, pp. 2498-2508, December 2004. DOI: 10.1056/NEJMoa033447.

[2] J. S. Perlmutter, "Assessment of Parkinson disease manifestations," Current protocols in neuroscience. Chapter. 10, Unit10.1, October 2009. DOI:10.1002/0471142301.ns1001s49.

[3] G. Ebersbach, C. Moreau, F. Gandor, L. Defebvre, and D. Devos, "Clinical syndromes: parkinsonian gait," Movement Disorder. vol. 28, Issue. 11, pp. 1552-1559, September 2013. DOI: 10.1002/mds. 25675.

[4] E. Abdulhay, N. Arunkumar, K. Narasimhan, and E. Vellaiappan, "Gait and tremor investigation using machine learning techniques for the diagnosis of Parkinson disease," Future Generation Computer Systems. vol. 83, pp. 366-373, June 2018. DOI: 10.1016/j.future.2018.02.009.

[5] Ö. F. Ertuğrul, Y. Kaya, R. Tekin, and M. N. Almall, "Detection of Parkinson's disease by Shifted One Dimensional Local Binary Patterns from gait," Expert Systems with Applications. vol. 56, pp. 156-163, September 2016. DOI: 10.1016/j.eswa.2016.03.018.

[6] L. Medeiros, H. Almeida, L. Dias, M. Perkusich, and R. Fischer, "A Gait Analysis Approach to Track Parkinson's Disease Evolution Using Principal Component Analysis," IEEE 29th International Symposium on Computer-Based Medical Systems (CBMS). pp. 48-53, June 2016. DOI: 10.1109/CBMS.2016.14.

[7] W. Schiehlen and D. García-Vallejo, "Walking dynamics from mechanism models to parameter optimization," Procedia IUTAM. vol. 2, pp. 199-211, 2011. DOI: 10.1016/j.piutam.2011.04.020

[8] D. Gouwanda and S. M. N. A. Senanayake, "Emerging Trends of BodyMounted Sensors in Sports and Human Gait Analysis," 4th Kuala Lumpur International Conference on Biomedical Engineering. vol. 21, pp. 715718, 2008. DOI: 10.1007/978-3-540-69139-6_178.
[9] H. Lee, S. J. Sullivan, and A. G. Schneiders, "The use of the dual-task paradigm in detecting gait performance deficits following a sports-related concussion: A systematic review and meta-analysis," Journal of Science and Medicine in Sport. vol. 16, no. 1, pp. 2-7, January 2013. DOI: 10.1016/j.jsams.2012.03.013.

[10] M. Alotaibi and A. Mahmood, "Improved Gait Recognition Based on Specialized Deep Convolutional Neural Network," Computer Vision and Image Understanding. vol. 164, pp. 103-110, November 2017. DOI: 10.1016/ j.cviu.2017.10.004.

[11] G. Qian, J. Zahang, and A. Kidane, "People Identification Using Floor Pressure Sensing and Analysis," IEEE Sensors Journal. vol. 10, pp. 1447 60, July 2010. DOI: 10.1109/JSEN.2010.2045158.

[12] M. J. Major, P. Raghavan, and S. Gard, "Assessing a low-cost accelerometer-based technique to estimate spatial gait parameters of lower-limb prosthesis users," Prosthetics and Orthotics International. vol. 40, no. 5, pp. 643-648, March 2015. DOI: 10.1177/0309364614568411.

[13] R. Camicioli, D. Howieson, S. Lehman, and J. Kaye," Talking while walking: The effect of a dual task in aging and Alzheimer's disease," Neurology. vol. 48, no.4, pp. 955-958, April 1997. DOI: 10.1212/WNL.48.4.955.

[14] S. M. Waechter et al. "The impact of dual tasking on cognitive performance in a Parkinson's disease cohort with and without freezing of gait: An EEG and behavioral based approach," 7th International IEEE/EMBS Conference on Neural Engineering (NER). pp. 1072-1075, April 2015. DOI: 10.1109/NER.2015.7146813

[15] P. Lorenzi et al. "Smart sensors for the recognition of specific human motion disorders in Parkinson's disease," 6th International Workshop on Advances in Sensors and Interfaces (IWASI). pp. 131-136, June 2015. DOI: 10.1109/IWASI.2015.7184973.

[16] F. Chollet, Keras “The python deep learning library”, [Online]. Available: https://keras.io/

[17] I. Goodfellow, Y. Bengio, and A. Courville, “Deep learning, ” MIT Press. Chapter. 2, pp. 326-415, 2016. ISBN: 978-0262035613.

[18] O. Costilla-Reyes, P. Scully, and K. B. Ozanyan, "Deep Neural Networks for Learning Spatio-Temporal Features From Tomography Sensors," IEEE Transactions on Industrial Electronics. vol. 65, no. 1, pp. 645-653, January 2018. DIO: 10.1109/TIE.2017.2716907.

[19] G, Yogev, N. Giladi, N. Peretz, S. Springer, E. S. Simon, and M. J. Hausdorff, "Dual tasking, gait rhythmicity, and Parkinson's disease: Which aspects of gait are attention demanding?" European Journal of Neuroscience. vol. 22, pp. 1248-1256, September 2005. DOI: 10.1111/j.1460-9568.2005.04298.

[20] M. J. Hausdorff, J. Lowenthal, T. Herman, L. Gruendlinger, C. Peretz, and N. Giladi, "Rhythmic auditory stimulation modulates gait variability in Parkinson's disease," European Journal of Neuroscience. vol. 26, pp. 2369-2375, October 2007. DOI: 10.1111/j.1460-9568.2007.05810.

[21] S. Frenkel-Toledo, N. Giladi, C. Peretz, T. Herman, L. Gruendlinger, and J. M. Hausdorff, "Treadmill walking as a pacemaker to improve gait rhythm and stability in Parkinson's disease," Movement Disorders. vol. 20, pp. 1109-1114, September 2005. DOI: 10.1002/mds.20507.

[22] O. Costilla-Reyes, P. Scully, and K. B. Ozanyan, "Temporal Pattern Recognition in Gait Activities Recorded With a Footprint Imaging Sensor System," IEEE Sensors Journal. vol. 16, no. 24, pp. 8815-8822, December 2016. DOI: 10.1109/JSEN.2016.2583260.

[23] D. P. Kingma and J. L. Ba, "Adam: a Method for Stochastic Optimization," $3 r d$ International Conference on Learning Representations (ICLR). 2015.

[24] S. Ruskaa, W. Hämäläinen, S. Kajava, M. Mughal, P. Matilainen, and J. Mononen, "Evaluation of the confusion matrix method in the validation of an automated system for measuring feeding behaviour of cattle," Behavioural Processe. vol. 148, pp. 56-62, March 2018. DOI: 10.1016/j.beproc.2018.01.004

[25] Y. Wu, P. Chen, X. Luo, M. Wu, L. Liao, S. Yang, and R.M. Rangayyan, "Measuring signal fluctuations in gait rhythm time series of patients with parkinson's disease using entropy parameters," Biomedical Signal Processing and Control. vol. 31, pp. 265-271, January 2017. DOI: org/10.1016/j.bspc.2016.08.02. 